Nota de investigación

\title{
Distribución y densidad de Rottboellia cochinchinensis (Lour.) Clayton en el estado de Morelos
}

\author{
Cid Aguilar Carpio ${ }^{1 \S}$ \\ Leobardo Díaz Corro ${ }^{2}$ \\ Juan Carlos Beltrán Mendoza ${ }^{2}$ \\ Gaudencio Millán Medina²
}

${ }^{1}$ IDAGRO S de RL de CV. Carretera Yautepec-Tlayacapan s/n, Col. Puente Pantitlán, Tlayacapan, Morelos. CP. 62545. ${ }^{2}$ Comité Estatal de Sanidad Vegetal del Estado de Morelos AC. Bajada Chapultepec núm. 23, Col. Chapultepec, Cuernavaca, Morelos. CP. 62450.

${ }^{\S}$ Autor para correspondencia: aguilar.cid@colpos.mx.

\section{Resumen}

En México, se puede observar un incremento en la introducción y diseminación de malezas cuarentenadas, las cuales, pueden causar daños económicos millonarios a la agricultura, por requerir medidas nuevas de combate en los cultivos. El objetivo del estudio fue determinar la distribución y densidad poblacional de Rottboellia cochinchinensis (zacate peludo) en el estado de Morelos y así conocer la época de mayor propagación con referencias a las condiciones ambientales y con ello evaluar el momento más oportuno para controlar o erradicar al zacate peludo, antes de que se pueda reproducir. El estudio se realizó del 01 de enero al 31 de diciembre de 2015, en las zonas agrícolas de los 33 municipios del estado de Morelos, donde fueron muestreados para identificar la presencia de $R$. cochinchinensis. Una vez identificada la existencia del zacate peludo en los municipios, cada mes se dio seguimiento a los municipios con presencia para conocer la densidad poblacional. Los municipios con la población más densa fueron Jantetelco, Jonacatepec, Tepalcingo y Xochitepec. La densidad de población más alta se presentó en los meses de julio y agosto después de observarse la mayor precipitación, por lo que es importante aplicar técnica de control sobre Rottboellia cochinchinensis antes del periodo mencionado entre los meses de mayo y junio.

Palabras clave: Rottboellia cochinchinensis, cuarentendas, maleza.

Recibido: septiembre de 2019

Aceptado: octubre de 2019 
En la actualidad existe un incremento del comercio y transporte entre países, lo que ha ocasionado un aumento en la introducción y diseminación de plantas exóticas, en algunos casos de forma no intencional. Una planta exótica se podrá considerar cuarentenaria por su historial como plaga y por las condiciones bióticas y abióticas que favorecen su establecimiento. Cada plaga tiene ciertos requerimientos de temperatura, humedad, suelo y altitud, que, de no cumplirse, dificultan o impiden su establecimiento en un nuevo territorio (Labrada, 2015).

En México, se puede observar un incremento en las malezas cuarentenadas originadas por el comercio con el exterior. Este tipo de malezas, pueden causar daños económicos millonarios a la agricultura, por requerir medidas nuevas de combate en cultivos u otros ambientes (Delgado et al., 2008).

El zacate peludo (Rottboellia cochinchinensis (Lour.) Clayton) conocido inicialmente como $R$. exaltata L., es una gramínea anual de autopolinización, erecta y está clasificado como una maleza cuarentenada ampliamente distribuida en los estados colindantes al Golfo de México (Campeche, Tabasco y Veracruz) se encuentran en los cultivos de arroz, algodón, maíz, sorgo y caña de azúcar, así como en potreros y plantaciones tropicales (mangos, cítricos, papaya) (Alves et al., 2003; Esqueda, 2005; Silva et al., 2009; Sánchez-Ken et al., 2012; Bundit et al., 2014; León et al., 2015). En México, esta especie fue colectada por primera vez en la zona de Escárcega, Campeche, en 1982 (Esqueda, 2005).

En el estado de Morelos se ha detectado la presencia de $R$. cochinchinensis (Sánchez-Ken y CerrosTlatilpa, 2016). Esta especie es considerada una de las 12 peores malezas que infestan a la caña de azúcar en el mundo y está clasificada como nociva por el Departamento de Agricultura de los Estados Unidos (Alves et al., 2003).

Puede causar pérdidas en el rendimiento de 20 a $70 \%$, dependiendo del cultivo que afecte (Contreras-Ramos et al., 2013). El uso de herbicidas ha sido un factor en la erradicación de esta especie; sin embargo, la equivocada época de aplicación ha permitido que el zacate peludo se propague, afectando a cultivos importantes (caña de azúcar, maíz y sorgo) en el estado de Morelos.

Por lo anterior, el objetivo del estudio fue determinar la distribución y densidad poblacional de $R$. cochinchinensis (zacate peludo) en el estado de Morelos y así conocer la época de mayor propagación con referencia a las condiciones ambientales y con ello determinar el momento más oportuno para controlar o erradicar al zacate peludo, antes de que se pueda reproducir.

El estudio se realizó del 01 de enero al 31 de diciembre de 2015, en las zonas agrícolas de los municipios del estado de Morelos, que se localiza geográficamente en la parte centro sur de México a $\operatorname{los} 19^{\circ} 08^{\prime}$ latitud norte, $18^{\circ} 19^{\prime}$ latitud sur, $98^{\circ} 38^{\prime}$ longitud este y $99^{\circ} 20^{\prime}$ longitud oeste, con una superficie de $4892.7 \mathrm{~km}^{2}$. El estado de Morelos presenta altitudes desde los 720 a los 5432 m, donde predomina el clima cálido subhúmedo (INEGI, 2013).

Los 33 municipios del estado de Morelos fueron muestreados para identificar la presencia de $R$. cochinchinensis; la identificación se realizó con base en la Guía de identificación de malezas Southern Weed Science Society (Elmore, 1990). Para el muestreo se seleccionaron 40 predios $(\approx$ $1000 \mathrm{~m}^{2}$ cada lugar) por municipio donde se esperaba encontrar la presencia del zacate peludo, que estuvieran cerca de cultivos agrícolas y donde creciera sin ser afectado por los agricultores. 
En cada predio se realizó un recorrido en forma de zig-zag que abarcó la mayor parte de la superficie, donde se seleccionaron 12 puntos al azar para realizar el muestreo. La densidad de población se cuantifico con un cuadrante metálico de 50 x $50 \mathrm{~cm}\left(0.25 \mathrm{~m}^{2}\right)$, y se contó el número de plantas de $R$. cochinchinensis presentes en el interior del cuadrante para después extrapolarlo a plantas por $\mathrm{m}^{2}$ (Trujillo et al., 2013).

Cada mes se dio seguimiento a los predios muestreados en los municipios con presencia para conocer la densidad poblacional. Los datos obtenidos se concentraron en una hoja de cálculo en el programa Excel®, donde se anotó el número de individuos de $R$. cochinchinensis presentes por mes y municipio, así como un promedio de todo el ano. La temperatura promedio y precipitación de los municipios donde se encontró la maleza cuarentenada se obtuvo de las estaciones pluviométricas pertenecientes a la red del Servicio Meteorológico Nacional y la CONAGUA.

En 19 de los 33 municipios del estado de Morelos, se encontró a R. cochinchinensis. La población más alta durante el año se observó en los municipios de Jantetelco, Jonacatepec, Tepalcingo y Xochitepec, los cuales presentaron una densidad de 172 a 184 plantas $\mathrm{m}^{-2}$ y la temperatura promedio que se presento fue de 27 a $24{ }^{\circ} \mathrm{C}$, así como una precipitación de entre 1624 a $1017 \mathrm{~mm}$ (Figura 1). A su vez, la menor densidad se registró en los municipios de Ayala, Cuautla, Tetecala y Yecapixtla, con una densidad de población de entre 104 y 112 plantas $\mathrm{m}^{-2}$; cabe señalar, que se ha determinado que $R$. cochinchinensis, incluso a densidades de 55 plantas $\mathrm{m}^{-2}$ puede reducir el rendimiento del cultivo de maíz hasta en 47\% (Strahan et al., 2000; León et al., 2015).

En la Figura 1 se observa la distribución de $R$. cochinchinensis en los municipios del estado de Morelos. Se puede apreciar que esta especie está adaptada a las condiciones ambientales donde predominan los climas cálidos subhúmedos, con temperaturas promedio de $21.5^{\circ} \mathrm{C}$. León et al (2015) indican que la temperatura base para que $R$. cochinchinensis germine es de $20^{\circ} \mathrm{C}$ y que, a temperaturas inferiores, esta especie no se propaga. Cabe señalar, que los municipios con mayor densidad de población de $R$. cochinchinensis colindan con el estado de Puebla, por lo que posiblemente se diseminó debido al flujo de maquinaria agrícola, agua, así como animales, desde Veracruz a Puebla y de ahí a Morelos (Esqueda, 2005).

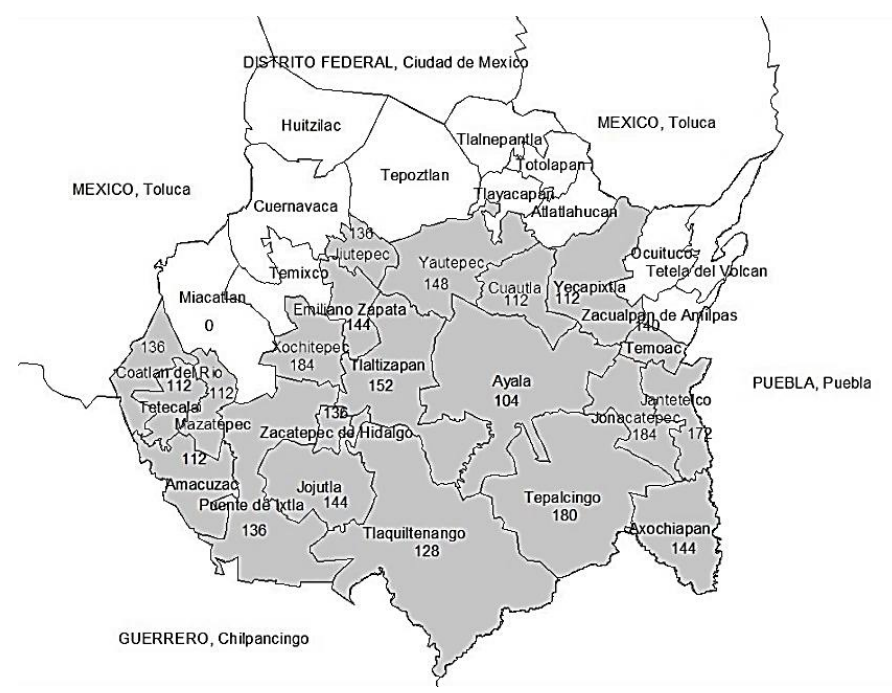

Figure 1. Distribución y densidad de población de Rottboellia cochinchinensis en el estado de Morelos (18 ${ }^{\circ} 44^{\prime} 51^{\prime}$ ' latitud norte, $99^{\circ}$ 04' 13" longitud oeste). Los municipios en color indican la presencia y los números la densidad de población promedio durante el año (plantas $\mathrm{m}^{-2}$ ). 
En cuanto a la densidad poblacional (Figura 2), de enero a marzo se observó una densidad que se mantuvo en un intervalo de 32 a 40 plantas por $\mathrm{m}^{2}$, ya que la ausencia de humedad afecto su propagación. La baja población a inicio de año se debió probablemente a que la maleza emergida en el mes de octubre presentó resistencia a la sequía (Bolfrey-Arku et al., 2011). En abril se presentaron aumentos en la densidad de población, en coincidencia con las primeras lluvias del temporal.

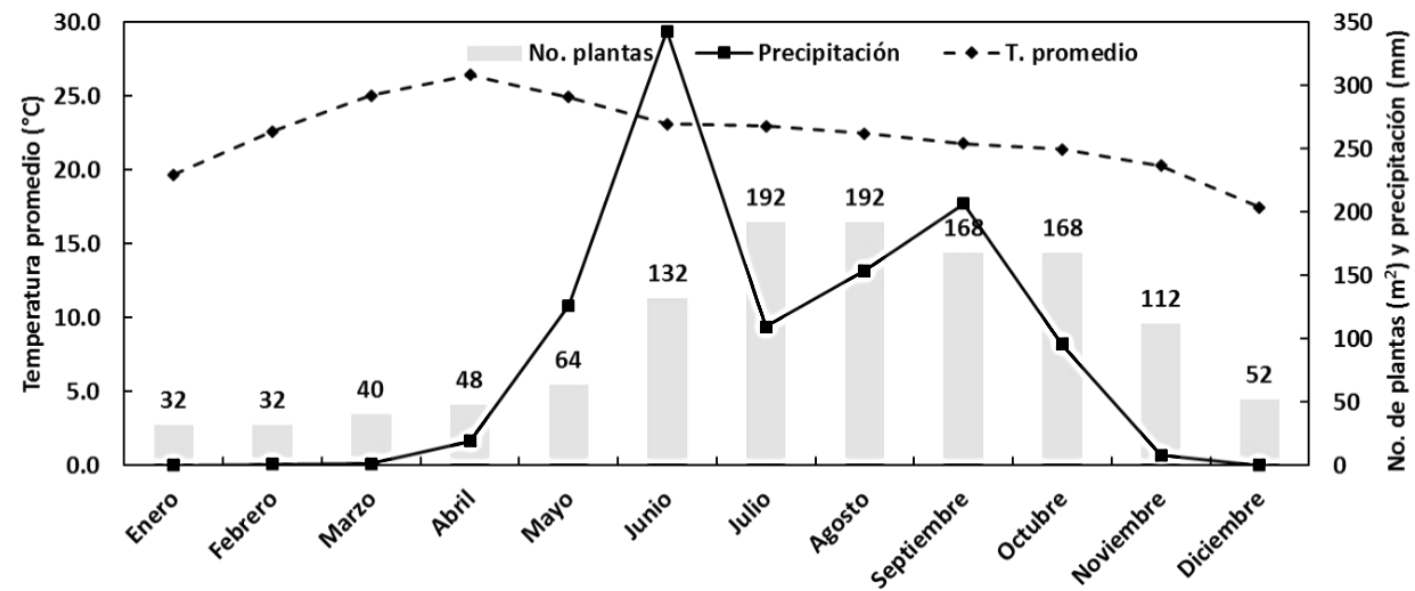

Figure 2. Densidad de población mensual promedio de $R$. cochinchinensis en las condiciones ambientales (precipitación y temperatura) del estado de Morelos.

La mayor precipitación se presentó en junio, esto favoreció la más alta densidad en julio, la cual se mantuvo hasta octubre, donde la precipitación empezó a disminuir. La baja humedad y temperatura durante los meses de noviembre y diciembre ocasiono una reducción en las densidades de población. Por otro lado, las poblaciones de $R$. cochinchinensis se desarrollaron en temperaturas que oscilaron entre 20 a $26{ }^{\circ} \mathrm{C}$ de enero a noviembre, siendo temperaturas óptimas para que se disemine el zacate peludo $\left(21.8^{\circ} \mathrm{C}\right)$, como lo indican Silva et al. (2009); Bolfrey-Arku et al. (2011); León et al. (2015).

\section{Conclusiones}

Los municipios del estado de Morelos con la población más densa fueron Jantetelco, Jonacatepec, Tepalcingo y Xochitepec. La densidad de población más alta se presentó en los meses de julio y agosto despues de observarse la mayor precipitación, por lo que es importante aplicar técnica de control sobre Rottboellia cochinchinensis antes del periodo mencionado entre los meses de mayo y junio.

\section{Literatura citada}

Alves, P. L.; Bachega, M. F.; Moro, J. R.; Lemos, M. V. F.; Alves, E. C. C.; Silva, M. A. S. and Moro, F. V. 2003. Identification and characterization of different accessions of itchgrass (Rottboellia cochinchinensis). Weed Science. 51(2):177-180. DOI: 10.1614/00431745(2003)051[0177:IACODA]2.0.CO;2. 
Bolfrey-Arku, G. E. K.; Chauhan, B. S. and Johnson, D. E. 2011. Seed germination ecology of itchgrass (Rottboellia cochinchinensis). Weed Sci. 59(2):182-187. DOI: 10.1614/WS-D10-00095.1.

Bundit, A.; Auvuvhanon, A. and Pornprom, T. 2014. Classification of population structure for allelopathic properties in itchgrass (Rottboellia cochinchinensis). Agrivita. 36(3):249-259.

Contreras-Ramos, S. M.; Rodríguez-Campos, J.; Saucedo-García, A.; Cruz-Ortega, R.; MacíasRubalcava, M. L.; Hernández-Bautista, B. E.; Dendooven, L.; Esqueda-Esquivel, V. A. and Anaya, A. L. 2013. Mutual effects of Rottboellia cochinchinensis and maize grown together at different densities. Agron. J. 105(6):1545-1554.

Delgado, M.; Ortiz, D. A. y Zambrano, A. 2008. Población de Rottboellia cochichinensis (Lour.) W. D. Clayton con resistencia cruzada al foramsulfuron + iodosulfuron. Agronomía Tropical. 58(2):175-180.

Elmore, D. C. 1990. Weed identification guide. Southern Weed Sciences Society. Champaign, IL. $250 \mathrm{p}$.

Esqueda, E. V. A. 2005. Efecto de herbicidas sobre plantas y semillas de Rottboellia cochinchinensis (Lour.) W. Clayton, en caña de azúcar. Agron. Mesoam. 16(1):45-50. DOI: 10.15517/am.v16i1.5181.

INEGI. 2013. Perspectiva estadística Morelos. Instituto Nacional de Estadística y Geografía. México, DF. 93 p.

Labrada, R. 2015. Revisión de la lista de malezas cuarentenadas de Ecuador. Revista La Técnica. 15(2):58-68. http://revistas.utm.edu.ec/index.php/latecnica/article/view/553/427.

León, R. G.; Izquierdo, J. and González-Adujar, J. L. 2015. Characterization and modeling of itchgrass (Rottboellia cochinchinensis) biphasic seedling emergence patterns in the tropics. Weed Sci. 63(3): 623-630. DOI: 10.1614/WS-D-14-00172.1.

Sánchez-Ken, J. G. y Cerros-Tlatilpa, R. 2016. Listado florístico de la familia Poaceae del estado de Morelos, México. Acta Botánica Mexicana. 116(3): 65-105. http://www.scielo.org.mx/pdf/abm/n116/2448-7589-abm-116-00065.pdf.

Sánchez-Ken, J. G.; Zita, P. G. de los A. y Mendoza, C. M. 2012. Catálogo de las gramíneas malezas nativas e introducidas de México. Primera Edición. SAGARPA, ASOMECIMA, AC, UNAM, CONACOFI. 433 p. https://www.researchgate.net/profile/GabrielSanchezKen/publication/256496385_Catalogo_de_malezas_gramineas_nativas_e_introducidas_de_ Mexico/links/02e7e5231f0711998a000000/Catalogo-de-malezas-gramineas-nativas-eintroducida s-de-Mexico.pdf.

Silva, C. E. B.; Parreira M. C.; Alves, P. L. y Pavani, M. C. 2009. Aspectos germinativos de capimcamaote (Rottboellia cochinchinensis). Planta Daninha. 27(2):273-281. DOI: 10.1590/S0100-83582009000200009.

Strahan, R. E., Griffin, J. L., Reynolds, D. B. and Miller, D. K. 2000. Interference between Rottboellia cochinchinensis and Zea mays. Weed Sci. 48(2):205-211. DOI: 10.1614/00431745(2000)048[0205:IBRCAZ]2.0.CO;2.

Trujillo, A. F. J.; Arias, R. R.; Sánchez, A. H. M. y Ramírez, Del A. M. 2013. Manual operativo de la campaña contra malezas reglamentadas. SAGARPA. SENASICA, 30 p. https://www.gob.mx/cms/uploads/attachment/file/108116/Manual_Operativo.p. 